\title{
Management of massive subcutaneous emphysema with a surgical tracheostomy
}

\author{
Report \\ Christopher SN Yeoh, Azman Mawaddah, Mat Baki Marina and Sani Abdullah
}

Department of Otorhinolaryngology, University Kebangsaan Malaysia Medical Center

\begin{abstract}
Introduction: Interventions for massive subcutaneous emphysema is rarely reported in the literature with lack of comparative studies and strong preferences over one treatment option compared to another. Tracheostomy is a conservative measure as compared to primary closure of tracheobronchial injury for the management of massive subcutaneous emphysema.

Case report: A 62-year gentleman who developed massive subcutaneous emphysema on the $12^{\text {th }}$ post-operative day following two laparoscopic abdominal surgeries and an exploratory laparotomy. A computer tomographic scan was performed showing a suspicious tenting of the trachea at right posterolateral wall at the level of T2 vertebra with locules of air seen anterolaterally to the trachea. A subsequent microlaryngobronchoscopy performed showed no defect in the subglottic region, nor tracheobronchial tree. He was successfully managed with a surgical tracheostomy whereby the emphysema showed dramatic resolution on the second postoperative day.

Conclusion: Surgical tracheostomy demonstrated a success in managing massive subcutaneous emphysema.
\end{abstract}

Key Words: Conservative, emphysema, tracheostomy, tracheobronchial injury.

Received: 18 November 2018, Accepted: 26 December 2018

Corresponding Author: Siu Ngee, Christopher Yeoh, MD, Department of Otorhinolaryngology - Head \& Neck Surgery, Hospital University Kebangsaan Malaysia Tel.: 0391455555, E-mail: chrisysnapp@gmail.com

ISSN: 2090-0740, November 2018, Vol.19, No. 3

\section{INTRODUCTION}

Tracheobronchial injury is an uncommon but potentially devastating problem and may be life threatening. With the improvement in hospital care and support, these injuries are encountered with increasing frequency. The majority of tracheobronchial injury is due to trauma and about $70 \%$ of patients die before admission and $21 \%$ of those reaching the hospital die within 2 hours $^{[1]}$. Iatrogenic injuries are often due to procedures such as esophagectomy, bronchoscopy, traumatic intubation and also tracheostomy ${ }^{[2]}$. The incidence of iatrogenic causes ranges from $0.5 \%$ to $0.19 \%{ }^{[3]}$.

Tracheobronchial injuries are often identified clinically during intraoperative period or during ventilation in the intensive care unit. They usually present with air leak during surgery and often easily recognized by the surgeon. Whereas in the intensive care unit setting, evidence of subcutaneous emphysema with evidence of pneumomediastinum may suggest tracheobronchial injury. Performing a computed tomography scan enables the clinician to localize the air leak and occasionally identify the lesion. As a standard practice, a computed tomography scan finding and a diagnostic microlaryngobronchoscopy are two important investigations to identify the location of the lesion for further intervention.
While subcutaneous emphysema is a recognized early complication of tracheostomy ${ }^{[2]}$, we would like to describe surgical tracheostomy as a treatment of massive subcutaneous emphysema.

\section{CASE REPORT}

A 62-year-old male with achalasia developed worsening dysphagia and gastroesophageal reflux disease. $\mathrm{He}$ had significant dilatation of the esophagus leading to esophageal failure. He underwent a thoracoscopic, laparoscopic total esophagectomy and neck anastomosis with feeding jejunostomy. On the 9th post-operative day, he developed severe abdominal pain associated with melaenic stools. A diagnosis of intra-abdominal bleeding was made, and he subsequently underwent an exploratory laparotomy for arrest of bleeding. He was kept intubated and ventilated in the intensive care unit following laparotomy due to sepsis. Three days later, the patient developed massive subcutaneous emphysema extending from the zygoma up to the chest at the level of $5^{\text {th }}$ intercostal space [Figure 1]. The laryngeal cartilage framework was palpable, and the trachea was central with subcutaneous emphysema over the neck. A computer tomographic scan was performed showed a suspicious tenting of the trachea at right posterolateral wall at the level 
of T2 vertebra with locules of air seen anterolaterally to the trachea [Figure 2]. A microlaryngobronchoscopy showed no defect in the subglottic region, trachea nor main bronchus. Following the findings, a tracheostomy was performed in the same setting. A wide skin incision was utilized with dissection of the subcutaneous tissue and strap muscles. The tracheal incision was performed on the third tracheal ring. The wound was closed loosely with dafilon $4 / 0$ to allow dissipation of the subcutaneous emphysema. Postoperatively, the patient showed significant resolution of the subcutaneous emphysema over a period of two days [Figure 3]. With the resolution of the subcutaneous emphysema, the patient was able to be weaned off the ventilator one day following surgical tracheostomy and tracheostomy was subsequently decannulated on the third post-operative day.

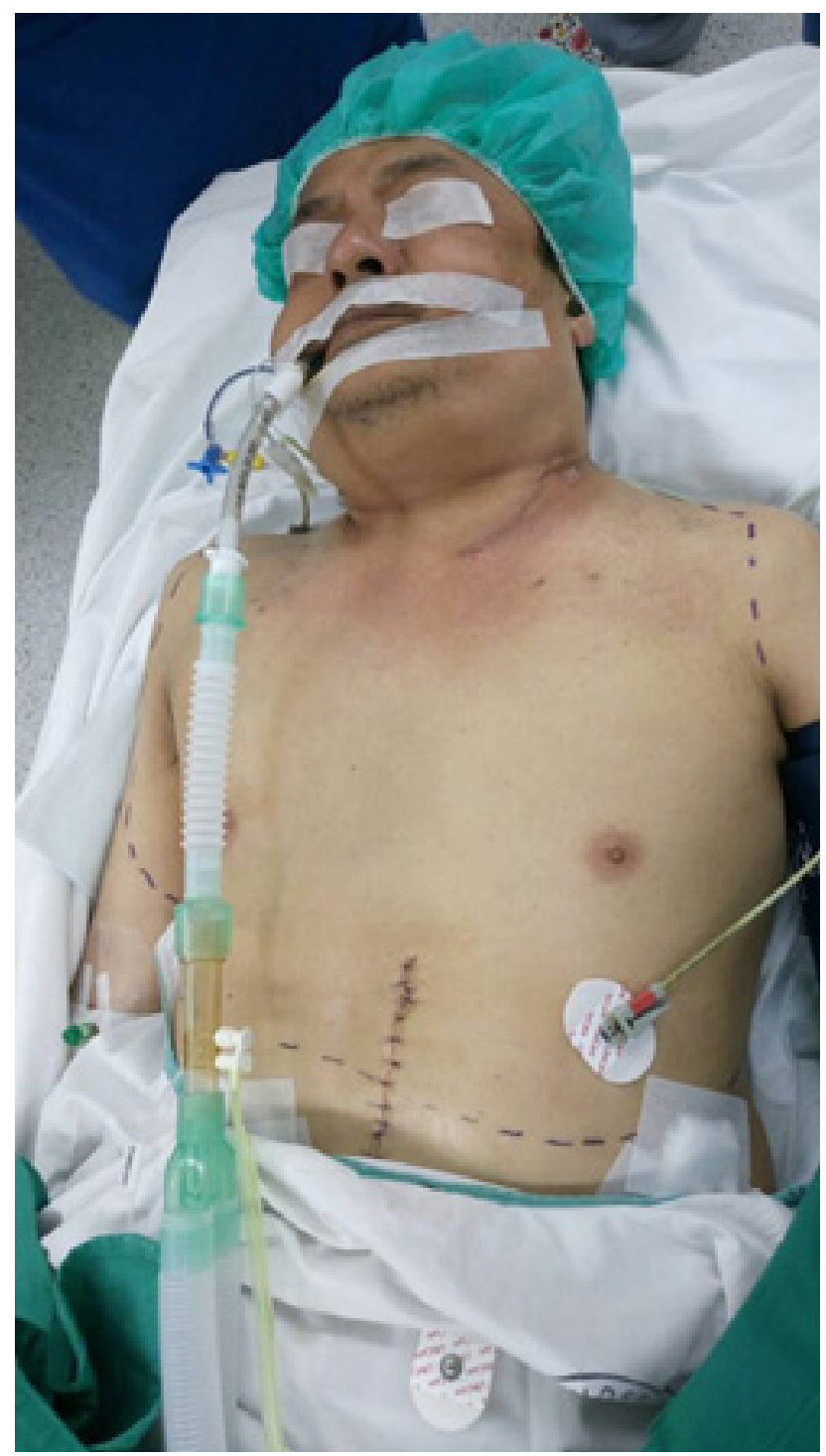

Fig. 1: Extensive subcutaneous emphysema extending from the zygoma up the chest

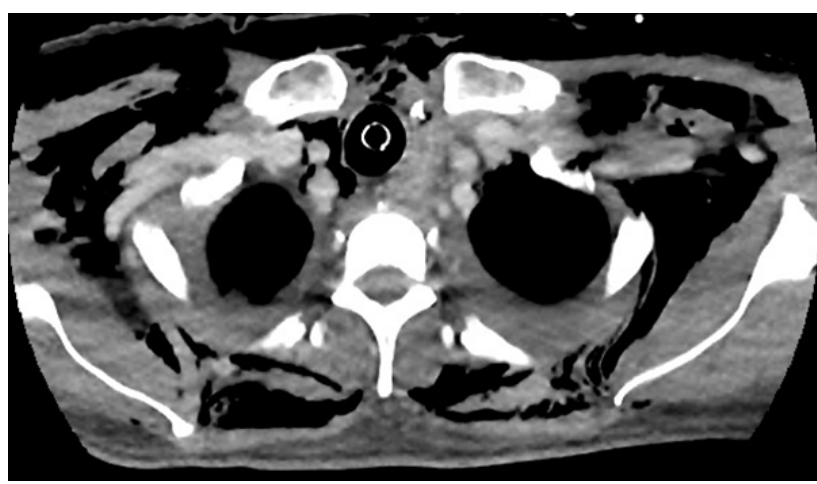

Fig. 2: show a suspicious tenting of the trachea at right posterolateral wall at the level of T2 vertebra with locules of air seen anterolaterally to the trachea

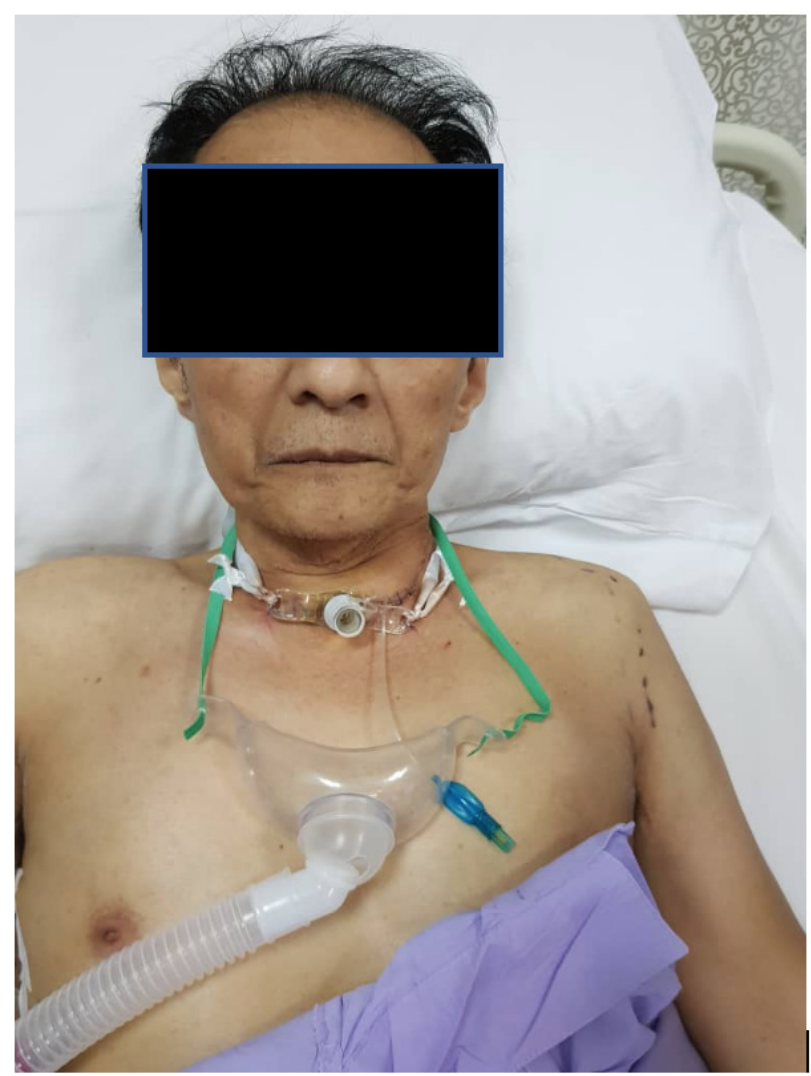

Fig. 3: Resolution subcutaneous emphysema at day 2 post tracheostomy

\section{DISCUSSION}

Cervical emphysema is a result of entry of air or gaseous material into the soft tissue planes within the neck. Various etiologies has been accounted for these occurrence and can be divided into iatrogenic and noniatrogenic. The common iatrogenic causes associated with tracheal injury are tracheostomy, traumatic endotracheal intubation, bronchoscopy and esophagectomy ${ }^{[2]}$. The 
incidence of such occurrence ranges from $0.05 \%$ to $0.19 \%{ }^{[3]}$. Non-iatrogenic causes are due to high impact accidents and many who suffers from it dies outside of the hospital despite the improvement in ambulatory services. It is said that iatrogenic cause of tracheobronchial injury is commoner than non-iatrogenic ${ }^{[4]}$. Early diagnosis of tracheal injury is important for good outcomes measures. How the patient presents, and the signs and symptoms is determined by the method and extent of the injury. Most of the time, small injuries occur unrecognized and heals spontaneous whereas some may lead to forming fistula or tracheal stenosis.

Esophagectomy is often divided into transhiatal approach and transthoracic approach. Transthoracic approach is usually combined with abdomino-thoracocervical approach through which the esophagus, surrounding tissue and lymph node is made resectable. The transthoracic approach is an abdominocervical where the esophagus is mobilized via the widened diaphragmatic hiatus and resected distally. The esophagus lies posteriorly to the tracheobronchial tree and is often at prone to injury during esophageal surgery. However, it is reported that tracheal injury during esophageal manipulation is as low with an average of $1 \%$ of total cases or even lower in experienced hands ${ }^{[5]}$.

Tracheal injury due to esophagectomy is often recognized immediately and visible within the operative field. Injuries arising from thermal injury or injury to the left main mainstem bronchus where lymph node dissection is carried out is often unrecognized till later ${ }^{[2]}$. Subcutaneous emphysema or mediastinal emphysema with persistent air leak is often seen and should raise concern of tracheal injury. On rarer occasions, pneumothorax or even tension pneumothorax and pneumoperitoneum in a case of tracheal injury have been reported ${ }^{[6]}$. Tracheal injury may also result in inability to ventilate the patient leading to a life-threatening event intraoperatively. A combination of hypoventilation with aspiration secondary to the leak may lead to atelectasis and pneumonia which worsens the condition of the patient.

Tracheal injury is often identified clinically intraoperatively or during ventilation in intensive care unit. Evidence to suggest tracheal injury is when the patient have difficulty ventilating or unknown source of subcutaneous emphysema. A chest radiograph is often helpful in diagnosis subcutaneous emphysema by evidence of striated lucencies within the soft tissue. If the emphysema is extensive involving the anterior chest wall outlining the pectoralis major muscle, it may give rise to a ginkgo leaf sign ${ }^{[7]}$. A computed tomography scan readily shows the anatomic location of the pockets of air amongst the soft tissue with evidence of extremely dark low attenuation areas within the subcutaneous space. Some studies describe air paralleling along the trachea as sign of tracheobronchial injury on a computed tomographic scan ${ }^{[8]}$.
A CT scan is able to show evidence of air leakage within the soft tissue and occasionally show its communication with the injury however, not always does the findings correspond with tracheoscopy findings. CT scans only have up to $85 \%$ sensitivity for detecting tracheal injury [8]. A direct laryngoscopy and tracheoscopy combined with computed tomography is the standard practice in assisting in identifying tracheal injury [9].

Tracheal injuries regardless of mechanism are life threatening events and surgical repair of these injuries have been described as the treatment of choice. This is based on the assumption that the injury may lead to mediastinitis or subsequent tracheal stenosis [1]. However, there are no comparative studies available in the literature to support this modality of choice. Moreover, primary repair carries a high risk of stricture formation and narrowing of the trachea [10]. Beiderlinden et al, contradicts this assumption as conservatively treated tracheal injuries has healed well without the feared complications [1]. Often conservative treatment is advocated for small tears over the trachea less than $2 \mathrm{~cm}$ in length [11]. Spontaneous recovery of these lesions has been increasingly reported after conservative treatment [12]. This is made possible by bridging the tracheal injury by intubating the patient or performing a tracheostomy distal to the site of injury [1]. Our experience with this case proves that it is not necessary to perform the tracheostomy distal to the site of injury and tracheostomy is a viable option, even for thoracic tracheal injuries which is the most likely cause in this case. Additionally, further intubating the patient will require prolonged ventilation and may lead to subsequent events. A tracheostomy is of better choice as this allows the patient to be easily weaned of the ventilator and also provide a pathway for breathing. The immobilization provided allows the lesion to heal spontaneously. A tracheostomy also helps to reduce the airway pressure within the trachea. This is proposed theoretically to prevent tension on the margins of the wound by reducing airway pressure during breathing, coughing or Valsalva maneuver hence promoting healing of the wounds [13]. The sealing of the lesion occurs naturally by tissue layers sliding and producing the same functional results as in a primary open surgical repair. Nonetheless, performing a tracheostomy also helps with clearance of tracheobronchial secretions without risk of further damaging the tracheal wall. More to that, by performing a tracheostomy as compared to extensive tracheal surgery, no tracheal cartilage is removed hence there is no shortening of the trachea and lessens the risk of tracheal stenosis [14]. Performing a wide incision along with loose closure of the tracheostomy wound allows the dissipation of air from the fascial planes and deep neck spaces. This helps to promote the resolution of the massive subcutaneous emphysema. This intervention, although similar to the previously described subcutaneous drain [15] or infraclavicular incision [16], offer better cosmesis to the patient. 


\section{CONCLUSION}

Interventions for massive subcutaneous emphysema is rarely reported in the literature with lack of comparative studies and strong preferences over one treatment option compared to another. Surgical tracheostomy, as demonstrated in this case was successful in treating massive subcutaneous emphysema due to iatrogenic thoracic tracheal injury following laparoscopic esophagectomy.

\section{REFERENCE}

1. Beiderlinden M,Adamzik M, Peters J. Conservative treatment of tracheal injuries. Anesth Analg. 2005 Jan;100(1):210-4.

2. Lui N, Wright C. Intraoperative Tracheal Injury. Thorac Surg Clin. 2015 Aug;25(3):249-54.

3. Leinung S, Mobius C, Hofinann HS, Ott R, Ruffert $\mathrm{H}$, Schuster $\mathrm{E}$ et al. Iatrogenic tracheobronchial ruptures - treatment and outcomes. Interact CardioVasc Thorac Surg 2006;5:303-6.

4. M Paraschiv. Iatrogenic tracheobronchial rupture. J Med Life. 2014 Sep;7(3):343-348.

5. Óvári A, Just T, Dommerich S, Hingst V, Böttcher A, Schuldt T, et al. Conservative management of post-intubation tracheal tears-report of three cases. J Thorac Dis. 2014 Jun;6(6):E85-91.

6. Orringer MB, Marshall $\mathrm{B}$, Iannettoni MD. Transhiatal esophagectomy: clinical experience and refinements. Ann Surg 1999;230:392-403.

7. Lal AB, Kumar N, Sami KA. Tension pneumoperitoneum from tracheal tear during pharyngolaryngoesophagectomy. Anesth Analg. 1995 Feb;80(2):408-9.

8. Medeiros BJ da C. Subcutaneous emphysema, a different way to diagnose. Rev Assoc Med Bras (1992). 2018 Feb;64(2):159-63.
9. Chen JD, Shanmuganathan K, Mirvis SE, et al Using CT to diagnose tracheal rupture. AJR Am J Roentgenol 2001;176:1273-80.

10. Hulscher JB, ter Hofstede E, Kloek J, Obertop H, De Haan P, Van Lanschot JJ. Injury to the major airways during subtotal esophagectomy: incidence, management, and sequelae. J Thorac Cardiovasc Surg. 2000 Dec;120(6):1093-6.

11. Carbognani P, Bobbio A, Cattelani L, Internullo E, Caporale D, Rusca M. Management of postintubation membranous tracheal rupture. Ann Thorac Surg 2004;77:406-9.

12. Miñambres E, Burón J, Ballesteros MA, Llorca J, Muñoz P, González-Castro A. Tracheal rupture after endotracheal intubation: a literature systematic review. Eur J Cardiothorac Surg. 2009 Jun;35(6):1056-62.

13. Carretta A, Melloni G, Bandiera A, Negri G, Voci C, Zannini P. Conservative and surgical treatment of acute posttraumatic tracheobronchial injuries. World J Surg. 2011 Nov;35(11):2568-74.

14. Marathe US, Tran LP. Pediatric neck trauma causing massive subcutaneous emphysema. J Trauma. 2006 Aug;61(2):440-3.

15. O'Reilly P, Chen HK, Wiseman R. Management of extensive subcutaneous emphysema with a subcutaneous drain. Respirol Case Rep. 2013 Dec;1(2):28-30.

16. Johnson CHN, Lang SA, Bilal H, Rammohan KS. In patients with extensive subcutaneous emphysema, which technique achieves maximal clinical resolution: infraclavicular incisions, subcutaneous drain insertion or suction on in situ chest drain? Interact Cardiovasc Thorac Surg. 2014 Jun;18(6):825-9. 